\title{
Active control based on finite element analysis
}

\author{
Li Hongying \\ Automotive Engineering institute, Jiangxi University of Technology, Nanchang 330098, China
}

Keywords: Intelligent structure; Active control; Finite element analysis; Simulation

\begin{abstract}
With the development of industry and advanced technology, vibration environment is becoming more and more complex. The traditional structural dynamics design is hard to meet the high reliability of the structure, so it's inevitable to seek new ways of vibration control. Active vibration control is the advanced technology in the current vibration engineering field, and is the integration of many subjects, such as dynamics, control, computer, testing technology and materials science, etc. This paper adopts finite element method commonly used in engineering for the analysis on plate structure modeling, and numerical simulation study on the active vibration control. Finally, calculation examples of the plate verify the effectiveness of previous work.
\end{abstract}

\section{Brief introduction}

With the development of industry and advanced technology, the scale of structure is becoming larger, and vibration environment is becoming more and more complex. The traditional structural dynamics design is hard to be applied. Structural vibration control has become one of the major topics of modern engineering technology, the intelligent control on suppression of structure deformation and disturbance has been highly valued in the engineering circle and theoretical circle.

Based on the need of external energy, structural vibration control methods are divided into three categories: passive control, active control and integrated passive and active control (also called hybrid control). Passive control is mainly the way that requires no external energy through adding damping materials into the structure, which is called damping processing. When the structure vibrates, strain of damping layer will transform the vibration energy into strain energy that can be dissipated as heat so as to restrain vibration, which is an effective way to solve the structural vibration control. The shortcoming of this method is limited damping effect to low frequency vibration, weak strain capacity to abrupt environment, and difficult to adapt to the complex and changing mechanical environment.

Active vibration control of structure is to reduce the response of the structure under environmental load by the use of external energy for control force, which is considered to be an effective method. In recent years, with the rapid development of material science, control, microelectronics and computer technology, especially the breakthrough of the study on new sensors and actuators, the new sensing materials and actuation materials are constantly applied to replace traditional sensors and actuators in the active vibration control of structure, which promotes the development of the new technology in structure design, and generates the brand new intelligent structure.

The finite element method is widely used in engineering practice at present; the existing mature application software, such as PATRAN/NASTRAN and ANSYS are easy for use and promotion. The finite element modeling and structural response analysis are simple and convenient by making use of existing software, and are free from the self-division of finite element and writing tedious 
programs; based on large and common structural finite element analysis software, integrate structure design, optimization, structure analysis and control design with simulation, complete in the same environment once for all, and truly realize the integration of structural control. This paper adopts finite element method commonly used in engineering for the analysis on plate structure modeling, and numerical simulation study on the active vibration control. Finally, calculation examples of the plate verify the effectiveness of previous work.

\section{Simulation study on vibration control}

The traditional design method of structural vibration control has always been to follow the flow shown in chart 1 , in which the design requirements of controller have a certain mathematical model; the certain mathematical model is very effective to simple structure; in the process of modeling for complex structure, simplified treatment of ignoring a few minor factors such as considering no higher order modes effect, reducing order, nonlinear link linearization and steady time varying parameters, and finally get the mathematical model suitable for control system design. The model is only an approximation of actual system, and different from previous model. However, the cost will increase through experimental method for structural vibration control, which apparently does not conform to the integrated design thought for modern structural control.

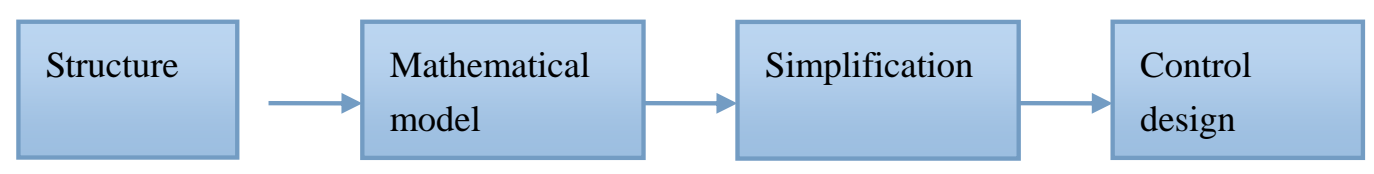

Figure. 1 Traditional control flow chart

With the development of finite element technology, the above problems have been improved a lot. Structural vibration control design technology based on finite element method directly designs for finite element model of structure rather than simplified mathematical model, as shown in chart 2. The basic idea is to make use of the finite element structure analysis technology, conduct control simulation experiments with finite element models replacing real object, determine control methods and optimize control parameters. The specific method is: firstly, build structural finite element model and load conditions; secondly, make structural dynamic response analysis, output feedback signal from analysis results and apply control signal through certain control algorithm in the structure; thirdly, analyze structural response, calculate and apply control signal; repeat the above process until the terminal conditions are satisfied. The whole process is just like the signal sensor, acquisition, processing, and actuator in the control experiment except that the actual response of the structure is replaced by finite element dynamic response analysis.

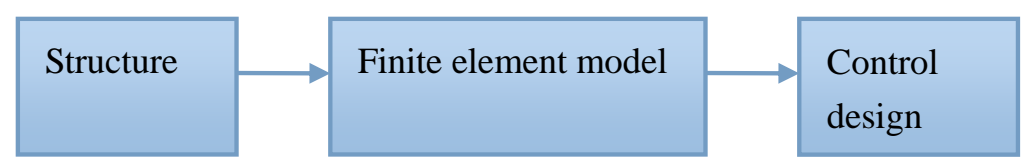

Figure. 2 Finite element controls 


\section{Finite element modeling}

As plate structure is a kind of simple and common structure, its modeling study is comparatively typical. Laminated piezoelectric plate is three-layer structure, with both linear elastic materials and nonlinear materials, so its finite element modeling and modal analysis are the key technologies. Generally, there are four kinds of piezoelectric formula for linear piezoelectric materials according to the different independent variables, of which the first and second formula are mostly used.

The first kind of piezoelectric equation expression is as follows, in which the independent variables are $\sigma$ standing for stress, and $\mathrm{E}$ standing for electric field intensity.

$$
\begin{gathered}
\{\mathrm{D}\}=[\mathrm{d}]\{\sigma\}+\left[\in^{\sigma}\right]\{\mathrm{E}\} \\
\{\varepsilon\}=\left[\mathrm{S}^{E}\right]\{\sigma\}+[d]^{t}\{\mathrm{E}\}
\end{gathered}
$$

In the first kind formula, $[\mathrm{d}]$ is piezoelectric strain constant matrix; $\{\mathrm{D}\}$ is electric displacement vector; $\left[\epsilon^{\sigma}\right]$ is dielectric constant matrix when stress $\sigma$ is zero or constant; $\{\varepsilon\}$ is strain vector; $\left[\mathrm{S}^{E}\right]$ is flexible flexibility constant matrix when electric field intensity E is zero or constant.

The second kind of piezoelectric equation expression is as follows, in which the independent variables are $\varepsilon$ standing for strain, and $\mathrm{E}$ standing for electric field intensity.

$$
\begin{aligned}
&\{\mathrm{D}\}=[\mathrm{e}]\{\varepsilon\}+\left[\epsilon^{\sigma}\right]\{\mathrm{E}\} \\
&\{\sigma\}=\left[\mathrm{C}^{\mathrm{E}}\right]\{\varepsilon\}-[\mathrm{e}]^{\mathrm{T}}\{\mathrm{E}\}
\end{aligned}
$$

In the second kind formula, $[\mathrm{e}]$ is piezoelectric stress constant matrix; $\left[\epsilon^{\sigma}\right]$ is dielectric constant matrix when strain $\varepsilon$ is zero or constant; [CE] is flexible stiffness constant matrix when electric field intensity $\mathrm{E}$ is zero or constant.

In the above two kind of piezoelectric formula, piezoelectric constant and flexible constant have the following relation:

$$
[\mathrm{e}]=[\mathrm{d}]\left[\mathrm{C}^{\mathrm{E}]}\right.
$$

For composite piezoelectric materials, the formula (2-1) and (2-2) can be expressed as:

$$
\left\{\begin{array}{l}
D_{1} \\
D_{2} \\
D_{3}
\end{array}\right\}=\left[\begin{array}{llllll}
0 & 0 & 0 & 0 & \mathrm{~d}_{15} & 0 \\
0 & 0 & 0 & \mathrm{~d}_{24} & 0 & 0 \\
d_{31} & \mathrm{~d}_{32} & \mathrm{~d}_{33} & 0 & 0
\end{array}\right]\left\{\begin{array}{l}
\sigma_{11} \\
\sigma_{22} \\
\sigma_{33} \\
\sigma_{23} \\
\sigma_{12} \\
\sigma_{12}
\end{array}\right\}+\left[\begin{array}{c}
\epsilon_{11}^{\sigma} \\
\epsilon_{22}^{\sigma} \\
\epsilon_{33}^{\sigma}
\end{array}\right]\left\{\begin{array}{l}
E_{1} \\
E_{2} \\
E_{3}
\end{array}\right\}
$$




$$
\left\{\begin{array}{l}
\varepsilon_{11} \\
\varepsilon_{22} \\
\varepsilon_{33} \\
\gamma_{23} \\
\gamma_{13} \\
\gamma_{12}
\end{array}\right\}=\left[\begin{array}{ccccccc}
\frac{1}{E_{1}^{P}} & -\frac{\mu_{12}^{\mathrm{P}}}{\mathrm{E}_{2}^{\mathrm{P}}} & -\frac{\mu_{13}^{\mathrm{P}}}{\mathrm{E}_{3}^{\mathrm{P}}} & 0 & 0 & 0 \\
-\frac{\mu_{21}^{\mathrm{P}}}{\mathrm{E}_{1}^{\mathrm{P}}} & \frac{1}{E_{2}^{P}} & -\frac{\mu_{23}^{\mathrm{P}}}{\mathrm{E}_{3}^{\mathrm{P}}} & 0 & 0 & 0 \\
-\frac{\mu_{31}^{\mathrm{P}}}{\mathrm{E}_{1}^{\mathrm{P}}} & -\frac{\mu_{32}^{\mathrm{P}}}{\mathrm{E}_{2}^{\mathrm{P}}} & -\frac{1}{\mathrm{E}_{3}^{\mathrm{P}}} & 0 & 0 & 0 \\
0 & 0 & 0 & \frac{1}{\mathrm{G}_{23}^{\mathrm{P}}} & 0 & 0 \\
0 & 0 & 0 & 0 & \frac{1}{\mathrm{G}_{23}^{\mathrm{P}}} & 0 \\
0 & 0 & 0 & 0 & 0 \frac{1}{\mathrm{G}_{23}^{\mathrm{P}}}
\end{array}\right]\left\{\begin{array}{c}
\sigma_{11} \\
\sigma_{22} \\
\sigma 33 \\
\tau_{23} \\
\tau_{13} \\
\tau_{12}
\end{array}\right\}+\left[\begin{array}{ccc}
0 & 0 & \mathrm{~d}_{31} \\
0 & 0 & \mathrm{~d}_{32} \\
0 & 0 & \mathrm{~d}_{33} \\
0 & \mathrm{~d}_{24} & 0 \\
\mathrm{~d}_{15} & 0 & 0 \\
0 & 0 & 0
\end{array}\right]\left\{\begin{array}{l}
E_{1} \\
E_{2} \\
E_{3}
\end{array}\right\}
$$

In the above formula, $E_{1}^{P}, E_{2}^{P}$, and $E_{3}^{P}$ are the elasticity modulus for piezoelectric modulus, is the shear modulus $\mu_{12}^{P}$ of Athens material are $G_{23}^{P}, G_{13}^{P}$ and $G_{12}^{P}$.

$$
\frac{\mu_{21}^{P}}{E_{1}}=\frac{\mu_{12}^{P}}{E_{2}} \quad \frac{\mu_{31}^{P}}{E_{1}}=\frac{\mu_{13}^{P}}{E_{2}} \quad \frac{\mu_{32}^{P}}{E_{1}}=\frac{\mu_{23}^{P}}{E_{2}}
$$

Since the advent of finite element technology in the 70s, many mechanical problems that is not solved or completely solved can be solved with the technology. Finite element technology has been developing so well for nearly 30 years that there seems to be no mechanical problems that can't be solved by finite element. Suitable finite element model shall be established for laminated plate unit analysis, and comparative analysis on the same issue adopting different unit when necessary. The weakness of this method is that the modeling of complex structure is very difficult, and the more accurate the model, the more degrees of freedom; the common workstations are hard to bear the resources consumption of the machine. For the simple structure of plate, all kinds of finite element programs such as Cosmosm, Nastran, Adina and Ansys can easily solve varied finite element analysis. The dynamics equations of system can be written in general form:

$$
[\mathrm{M}]\{\mathrm{u}\}+[\mathrm{C}]\{\mathrm{u}\}+[\mathrm{K}]\{\mathrm{u}\}=\{\mathrm{F}\}
$$

Cantilever $(10 \mathrm{~mm} * 5 \mathrm{~mm} * 1 \mathrm{~mm})^{(100 \mathrm{~mm} \times 5 \mathrm{~mm} \times 1 \mathrm{~mm})}$, top and bottom surface with Piezoelectric Transducer (PZT) are sensors and actuators, as shown in chart 3. The material constant of beam: elasticity modulus $E_{p}=7.05 * 10^{10} \mathrm{~N} / \mathrm{m}^{2}$, Poisson ratio $\mu_{k}=0.3$, mass density $\rho=2700 \mathrm{~kg} / \mathrm{m}^{2}$, supposing the model of PZT sensors and actuators is the same as the one for physical experiment, the thickness $\mathrm{h}=1 \mathrm{~mm}$, mass density $\rho=2600 \mathrm{~kg} / \mathrm{m}^{2}$, elasticity modulus $E_{p}=6.3 * 10^{10} \mathrm{~N} / \mathrm{m}^{2}, \mu_{k}=0.3$ 


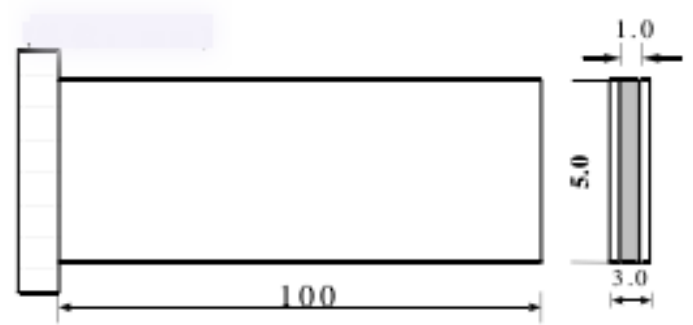

Figure. 3 Geometric model

Apply pulse with $1 \mathrm{~N}$, and action time 0.001 second on the point $\mathrm{A}$ of beam-end; the total mass matrix and stiffness matrix of the beam can be calculated by NASTRAN analysis. Lead the total mass matrix and stiffness matrix into MATLAB, and make simulating calculation in SIMULINK.

\section{Conclusion}

Vibration control experimental devices adopt xPC target real-time system based on MATLAB; the composition of control system is shown in chart 5-2, which mainly includes sensor, preamplifier, A/D converter, computer, D/A converter, driving power and actuators. Overall physical devices shown in chart 5-3, includes 2 sets of PC computer, 1 piece of A/D data collection card of 8-channel PCL-818L(integrated D/A conversion card), 1 set of power amplifier of 790 series AVC ((piezoelectric power supply), 1 set of sensing signal regulator of the PCI-481A model.

xPC target can be operated on one or two machines. For convenience of debugging program, the experimental devices in the paper adopt the mode of operating on two machines, namely host machine and target machine. In XPC target environment, the PC installed the software MATLAB, Simulink and Stateflow is the host machine; establish model with Simulink model and Stateflow chart and make non real-time simulation. Then make use of code generator like RTW and Stateflow and the $\mathrm{C}$ compiler for generating executable code and run it on the second compatible PC in real time. When the control system is in real-time control, only the target machine as controller sends control order; the host machine generates executable codes for the convenience of debugging program, and downloads it into the target machine. Based on the research of the piezoelectric plate unit, establish initiative structure model using commercial finite element software PATRAN/NASTRAN, build finite element modeling for structure and make varied dynamics analyses. The modeling method is effective according to the results in the paper.

On the premise of meeting the engineering accuracy, transform the coordinate of dynamic equation, adopt low order mode as vector base, truncate and decouple the high order mode. Study proportional feedback control method according to decoupling equation and the results of finite element software analysis; based on LQR (quadratic linear optimal control) independent modal control method, determine the gain of active structural control and control the vibration of the structure. In order to verify the validity of the above vibration control method, combined with a numerical example and experiment, determine the response of the active structure control before and after and make the curve. According to the simulation results and experimental results, the active control adopting piezoelectric structure is effective to the structural vibration suppression. The paper makes modal analysis on the active structure, and carries numerical simulation and experiment on active vibration suppression. 


\section{Acknowledgements}

This work was financially supported by the key subject building project (vehicle engineering) of Jiangxi University of Technology.

\section{Reference}

[1]Vlasov Y A, O'Boyle M, Hamann H F, et al. Active control of slow light on a chip with photonic crystal waveguides[J]. Nature, 2005, 438(7064): 65-69.

[2]Fuller C C, Elliott S, Nelson P A. Active control of vibration[M]. Academic Press, 1996.

[3]Handforth A, DeGiorgio C M, Schachter S C, et al. Vagus nerve stimulation therapy for partial-onset seizures A randomized active-control trial[J]. Neurology, 1998, 51(1): 48-55.

[4]Balas M J. Active control of flexible systems[J]. Journal of Optimization theory and Applications, 1978, 25(3): 415-436.

[5]Bauer M S, Mitchner L. What is a "mood stabilizer"? An evidence-based response[J]. 2014.

[6]Li P, Lam J, Cheung K C. Multi-objective control for active vehicle suspension with wheelbase preview[J]. Journal of Sound and Vibration, 2014, 333(21): 5269-5282.

[7]Yıldız A S, Sivrioğlu S, Zergeroğlu E, et al. Nonlinear adaptive control of semi-active MR damper suspension with uncertainties in model parameters[J]. Nonlinear Dynamics, 2014: 1-14.

[8]Kopperdahl D L, Aspelund T, Hoffmann P F, et al. Assessment of incident spine and hip fractures in women and men using finite element analysis of CT scans[J]. Journal of Bone and Mineral Research, 2014, 29(3): 570-580.

[9]Karimi A, Navidbakhsh M, Razaghi R. Dynamic simulation and finite element analysis of the human mandible injury protected by polyvinyl alcohol sponge[J]. Materials Science and Engineering: C, 2014, 42: 608-614.

[10] Benazzi S, Grosse I R, Gruppioni G, et al. Comparison of occlusal loading conditions in a lower second premolar using three-dimensional finite element analysis[J]. Clinical oral investigations, 2014, 18(2): 369-375. 